\title{
Adaptive Control of Single-Input, Single-Output Linear Systems
}

\author{
ARIE FEUER, MEMBER, IEEE, AND A. STEPHEN MORSE, SENIOR MEMBER, IEEE
}

\begin{abstract}
A procedure is presented for designing parameter-adaptive control for a single-input, single-output process admitting an essentially unknown but fixed linear model, so that the resulting closed-loop system is globally stable with zero steady-state tracking error between the output of the process and the output of a prespecified linear reference model. The adaptive controller is a differentiator-free dynamical system forced only by the process input and output, as well as by a reference input.
\end{abstract}

\section{INTRODLCTION}

$\mathbf{T}$ HERE ARE many examples of physical processes which require feedback control syestems capable of functioning at a number of different process operating points. In some instances, the parameters of the linearized process model upon which closed-loop control is based assume such a wide range of values during process operation that a single, fixed-parameter control system proves inadequate to regulate the process. In such cases parametric changes are often dealt with by using several fixedparameter controls and an appropriate switching logic; alternatively, in some cases model parameter values are precomputed and stored as functions of operating point, and a single control system with gains functionally dependent on stored model parameters is used. If the number of process model parameters is large, if the parameters cannot be computed with sufficient accuracy or if "tight" control is required to meet rigid specifications, neither type of control system may be capable of providing adequate regulation.

A promising alternative, potentially applicable in situations such as these, is a parameter-adaptive control system. Roughly speaking, a parameter-adaptive system is an adaptive control system with the capability of adjusting its own parameters to compensate for the slow but significant changes in process characteristics resulting from process transfer from one operating point to another. The idea of a parameter-adaptive system is, of course, not new [1]-[3]. Nevertheless, it is fair to say that the basic principles governing the design and operation of such systems are only now just beginning to be understood.

Manuscript received April 25, 1977; revised September 19, 1977 and March 8, 1978. Paper recommended by R. V. Monopoli, Past Chairman of the Adaptive, Learning Systems, Pattern Recognition Committee. This work was supported by the United States Air Force Office of Scientific Research under Grant 77-3176.

The authors are with the Department of Engineering and Applied Science, Yale University, New Haven, CT 06520.

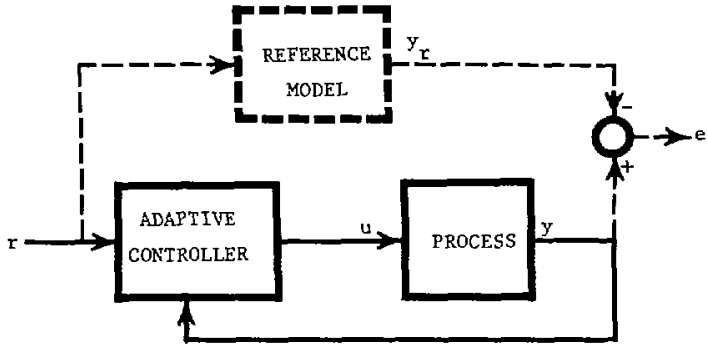

Fig. 1. Parameter-adaptive sytstem.

In this paper we consider the problem of designing a parameter-adaptive control system for a single-input, single-output process admitting an essentially unknown but fixed linear model, so that for any reference input $r(t)$, the tracking error $e(t)$ between the output $y(t)$ of the resulting controlled system (Fig. 1) and the output $y_{r}(t)$ of a prespecified linear reference model is regulated to zero asymptotically. We assume that only the process input $u(t)$ and output $y(t)$ can be measured (but not the process model state) and we require the controller to be a differentiator-free dynamical system realizable with conventional analog components.

Although a great many parameter-adaptive controllers have been proposed in the literature, only under very restrictive assumptions have any actually been shown to result in stable closed-loop systems. For example, in [3] Parks puts forth the idea of Lyapunov redesign to achieve stable adaptive operation, but the stability analysis given there is incomplete and the overall approach is limited to process transfer functions of relative degree one. In [4], Áström and Wittenmark propose a parameter-adaptive system consisting of an on-line recursive parameter identifier and a minimum-variance control law generator; the asymptotic properties of such "self-tuning regulators" have recently been examined in [5], [6], but global stability of these systems has not yet been established. In [7], Monopoli suggests an alternative configuration (a simplification of which is used in this paper) based on the important observation that under certain conditions it is not necessary to separately identify process model parameters and control feedback gains; but the arguments in [7] concerning stability contain errors and do not justify the paper's main claims [8]. In addition to these references there are numerous others dealing with parameter-adaptive control, but for one reason or another none apparen- 
tly give an adequate answer to the following fundamental question: Do parameter-adaptive controllers which yield globally stable closed-loop systems actually exist for single-input, single-output linear processes?

The purpose of this paper is to provide an affirmative answer to this question by presenting what we believe is the first parameter-adaptive control configuration, applicable to a reasonably large class of linear process models, which is known to result in a globally stable closed-loop system in which all signals and gains are guaranteed to remain bounded. The proposed controller requires no differentiators and can be realized with integrators, summers, gains, and multipliers. The only assumptions made about the process are that it admits a transfer function model with left-half plane zeros, and that: 1) an upper bound for the transfer function's "dimension," 2) the "relative degree" of the transfer function, and 3) the sign of the transfer function's "gain" are known.

In Section I the general structure of the controller is discussed as are the crucial process model assumptions upon which it is based; an interpretation of this structure in state-space terms reveals that the function of the controller is, in essence, to adaptively shift one subset of process model poles to prescribed locations, while adaptively cancelling process transfer function zeros with the others. Detailed descriptions of the control parameter adjustment law and the auxiliary control signal needed to guarantee system stability are given in Section II. Finally in Section III it is shown that application of the controller to any process satisfying the assumptions of Section I results in a globally stable closed-loop system which follows a prespecified linear reference model with zero steady-state output tracking error.

\section{Notation}

In the sequel, prime denotes transpose. If $M(t)$ and $N(t)$ are two $m \times n$ matrices of time functions, we write $M=N$ $(\epsilon)$ if each element of the matrix $M-N$ is a linear combination of decaying exponentials. If $\alpha(s)$ is a polynomial, $(\alpha(s))^{\circ}$ denotes its degree. The relative degree of a rational function $\alpha(s) / \beta(s)$, written $(\alpha(s) / \beta(s))^{\circ}$, is the integer $(\beta(s))^{\circ}-(\alpha(s))^{\circ}$. The zero-state output response of a linear system with input $f(t)$ and transfer function $\alpha(s) / \beta(s)$ is sometimes written as $(\alpha / \beta) f(t)$. A dynamical system of the form $\dot{x}=g(x, r)$ with piecewise-continuous input $r(t)$ is smooth if $g(\cdot)$ is a continuous function of its arguments; such a system is globally stable if for each initial time $t_{0} \geqslant 0$ and state $x_{0}$, the state response $x\left(x_{0}, t_{0}, t\right)$ exists for $t \geqslant t_{0}$ and the Euclidean norm $\| x\left(x_{0}, t_{0}, t \|\right.$ is bounded by a finite constant not depending on $t_{0}$. In the special case of a linear system, stable means asymptotically stable (i.e., all system eigenvalues are in the open left-half plane.) Similarly, a polynomial transfer matrix or square matrix is stable if its zeros, poles, or eigenvalues, respectively, lie in the open left-half plane.

\section{System Structure}

It is perhaps most natural to think of a parameter-adaptive controller as a system consisting of two distinct subsystems-one which dynamically generates asymptotic estimates $\hat{p}(t)$ and $\hat{x}(t)$ of process model parameters $p$ and state $x(t)$ respectively, the other which generates an estimate $\hat{f}(t)$ of desired feedback gains $f$ as a function of estimated model parameters $\hat{p}(t)$. However, in spite of its intuitive appeal, this particular configuration is difficult to analyze and at present is not well understood. There are at least three reasons for this.

1) Known results [9]-[11] characterizing the behavior of dynamic identifiers (e.g., adaptive observers) are not directly applicable, since such results usually require all identifier inputs to be bounded; in the present situation, neither the process input $u$ nor output $y$ can be assumed bounded a priori, since the identifier is in feedback with the process.

2) The relationship between process model parameters $p$ and desired feedback gains $f$ is typically a complicated nonlinear function $f(p)$. Roughly speaking, this is because the type of parameterized model which is amenable to on-line identification and state estimation is a linear system which is observable for all values of its free parameters, whereas the type of parameterized model which might conceivably yield a linear relationship between its parameters and desired feedback gains is one which must remain controllable for all values of its free parameters.

3) $f(p)$ is usually not well-defined at those points in parameter space at which the parameterized model used for identification is not controllable; thus, if the control generator shown in Fig. 2 is a memoryless realization of this function, then the possibility of an unbounded gain vector $\hat{f}$ cannot be ruled out [10].

By adopting a somewhat different concept of a parameter-adaptive system than that shown in Fig. 2, it is possible to avoid the preceding problems. The key idea is to generate a feedback control without utilizing distinct estimates of desired feedback gains $f$ and process model state $x(t)$. In the sequel, the general structure of such an adaptive system will be described.

Our basic assumption is that the relationship between the process input $u$ and output $y$ can be modeled by a canonical (i.e., controllable and observable) linear system

$$
\Sigma_{p}:\left\{\begin{array}{l}
\dot{x}_{p}(t)=A_{p} x_{p}(t)+b_{p} u(t) \\
y(t)=c_{p} x_{p}(t)
\end{array}\right\}
$$

with strictly proper transfer function

$$
T_{p}(s)=g_{p} \frac{\alpha_{p}(s)}{\beta_{p}(s)}
$$

where $g_{p}$ is a constant gain and $\alpha_{p}(s)$ and $\beta_{p}(s)$ are coprime monic polynomials. For reasons to be made clear in the sequel, we make the following 


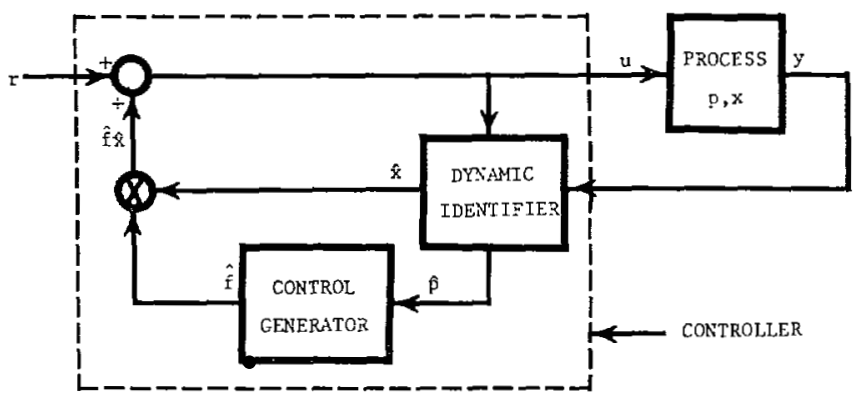

Fig. 2. A parameter-adaptive system with separate identification and control.

\section{Process Model Assumptions}

1) $\alpha_{p}(s)$ is a stable polynomial.

2) The following data are known:

a) The sign of $g_{p}$;

b) An integer $n \geqslant\left(\beta_{p}(s)\right)^{\circ}$;

c) The relative degree $n^{*} \equiv\left(\alpha_{p}(s) / \beta_{p}(s)\right)^{\circ}$.

The principal function of the adaptive controller shown in Fig. 1 is to force the process output $y$ to approach and track the output $y_{r}$ of a prespecified linear reference model. The reference model is a stable, canonical linear system

$$
\Sigma_{r}:\left\{\begin{array}{l}
\dot{x}_{r}(t)=A_{r} x_{r}(t)+b_{r} r(t) \\
y_{r}(t)=c_{r} x_{r}(t)
\end{array}\right\}
$$

with strictly proper transfer function $T_{r}(s)$ and bounded, piecewise-continuous, reference input $r(t)$. To determine what else is required of $\Sigma_{r}$ for zero tracking error to be possible, let us note that if the adaptive controller shown in Fig. 1 were replaced by any linear dynamical (i.e., differentiator-free) compensator $\Sigma$ with reference input $r$, measured input $y$ and output $u$, and if $T_{\Sigma}$ were the resulting closed-loop transfer function from $r$ to $y$, then the relative degree of $T_{\Sigma}$ would not be less than $n^{*}$. Indeed, this fundamental constraint on $T_{\Sigma}$ can be relaxed only by incorporating differentiators in $\Sigma$. Clearly, any reference model not respecting this constraint must involve some form of differentiation. Since we have stipulated that our adaptive controller be differentiator-free, we must require that $\left(T_{r}(s)\right)^{\circ} \geqslant n^{*}$.

The process model assumptions imply that the process can be represented in an especially useful way. To describe this representation, let $\alpha(s), \beta(s)$ and $\gamma(s)$ be any three polynomials which have been selected with knowledge of $n$ and $n^{*}$ so that
a) $\quad \alpha(s), \beta(s)$ and $\gamma(s)$ are monic and stable.
b) $\alpha(s)$ and $\beta(s)$ are coprime and $(\alpha(s) / \beta(s))^{\circ}=n^{*}$.
c) $\alpha(s)$ divides $\gamma(s)$ and $(\gamma(s))^{\circ} \geqslant n-1$.

It will be shown in the sequel that if $\alpha(s), \beta(s)$ and $\gamma(s)$ are so defined, then there must exist polynomials $\delta_{u}(s)$ and $\delta_{y}(s)$ such that

$$
y(t)=g_{p} \frac{\alpha(s)}{\beta(s)}\left(u(t)-\frac{\delta_{u}(s)}{\gamma(s)} u(t)-\frac{\delta_{y}(s)}{\gamma(s)} y(t)\right)
$$

where $\delta_{u}(s) / \gamma(s)$ and $\delta_{y}(s) / \gamma(s)$ are strictly proper and proper transfer functions, respectively. To understand the role of this representation, which has its origin in [7], first observe that (4) implies that the output tracking error

$$
e(t)=y(t)-y_{r}(t)
$$

can be written as

$$
\begin{array}{r}
e(t)=g_{p} \frac{\alpha(s)}{\beta(s)}\left(u(t)-\frac{\delta_{u}(s)}{\gamma(s)} u(t)-\frac{\delta_{y}(s)}{\gamma(s)} y(t)\right. \\
\left.-\frac{1}{g_{p}} T(s) r(t)\right)
\end{array}
$$

where $T(s) \equiv(\beta(s) / \alpha(s)) T_{r}(s)$; the assumptions on $T_{r}(s)$ (i.e., stability and $\left.\left(T_{r}(s)\right)^{\circ} \geqslant n^{*}\right)$, together with the constraints on $\alpha(s)$ and $\beta(s)$ dictated by (3a) and (3b) guarantee that $T(s)$ is a stable, proper transfer function.

Next, observe that since $\delta_{u}(s) / \gamma(s)$ and $\delta_{y}(s) / \gamma(s)$ are strictly proper and proper transfer functions, respectively, it is possible to write

$$
\frac{\delta_{u}(s)}{\gamma(s)}=\sum_{i=1}^{n_{\gamma}} k_{i} \frac{\gamma_{i}(s)}{\gamma(s)}
$$

and

$$
\frac{\delta_{y}(s)}{\gamma(s)}=\sum_{i=1}^{n_{\gamma}} k_{n_{y}+i} \frac{\gamma_{i}(s)}{\gamma(s)}+k_{2 n_{\gamma}+1}
$$

where the $k_{i}$ are constants, $n_{\gamma}=(\gamma(s))^{\circ}$, and $\left\{\gamma_{1}(s), \cdots, \gamma_{n_{1}}(s)\right\}$ is any preselected basis for the vector space of polynomials of degree less than $n_{\gamma}$ (e.g., $\gamma_{i}(s)=$ $\left.s^{i-1}\right){ }^{1}$ The implication of these expressions is that it is now possible to rewrite (6) as

$$
e(t)=g_{p} \frac{\alpha(s)}{\beta(s)}\left(u(t)-\theta^{\prime}(t) k\right)
$$

where $k \equiv\left[k_{1}, k_{2}, \cdots, k_{2 n+1}, 1 / g_{p}\right]$ is a vector of unknown constants; $\theta(t) \equiv\left[\theta_{1}(t), \theta_{2}(t), \cdots, \theta_{2 n_{2}+2}(t)\right]^{\prime}$ is a vector of known sensitivity functions obtained by passing $u, y$, and $r$ through the stable, canonical, linear system

$$
\Sigma_{\theta}:\left\{\begin{array}{c}
\dot{x}_{\theta}(t)=A_{\theta} x_{\theta}(t)+b_{u} u(t)+b_{y} y(t)+b_{r} r(t) \\
\theta(t)=C_{\theta} x_{\theta}(t)+d_{y} y(t)+d_{r} r(t)
\end{array}\right\}
$$

with transfer matrix $T_{\theta} \equiv$ block diag $\left\{T_{u}, T_{y}, T\right\}$, where $T_{u}=\left[\gamma_{1} / \gamma, \cdots, \gamma_{n_{i}} / \gamma\right]^{\prime}$ and $T_{y}=\left[\gamma_{1} / \gamma, \cdots, \gamma_{n_{1}} / \gamma, 1\right]^{\prime}$.

The expression for $e(t)$ in (7) shows that if $k$ were known, then zero-output tracking error could be achieved

${ }^{\text {IIf }}(\gamma(s))^{\circ}>n-1$, then $\delta_{y}(s) / \gamma(s)$ turns out to be strictly proper [cf Proposition 1]; thus, in this case $k_{2 n_{r}+1}=0$. 
(asymptotically) by setting $u(t)=\theta^{\prime}(t) k$. Since $k$ is not known, what we shall do instead is to set

$$
u(t)=\theta^{\prime}(t) \hat{k}(t)+\bar{u}(t)
$$

where $\hat{k}(t)$ is a suitable defined estimate of $k$ and $\bar{u}(t)$ is an auxiliary signal whose sole function is to guarantee system stability. In Section II we explain how to select $\hat{k}(t)$ and $\bar{u}(t)$ so that for any reference input $r(t)$, all system signals are bounded and $e(t) \rightarrow 0$ as $t \rightarrow \infty$.

The structure of the control law (9) is motivated primarily by the expression for $e$ in (7) which, in turn, is a consequence of (4). To establish the validity of (4), first note that since $\gamma(s)$ is a stable polynomial, $y(t)$ and $u(t)$ will satisfy (4) just in case $y(t)$ is a solution to the differential equation

$$
\beta(s) \gamma(s) y(t)=g_{p} \alpha(s)\left(\left(\gamma(s)-\delta_{u}(s)\right) u(t)-\delta_{y}(s) y(t)\right)
$$

Since process model assumption 1) asserts that $\alpha_{p}(s)$ is stable, (1) will imply (10) if and only if

$$
\frac{\alpha_{p}(s)}{\beta_{p}(s)}=\frac{\alpha(s)\left(\gamma(s)-\delta_{u}(s)\right)}{\gamma(s) \beta(s)+g_{p} \alpha(s) \delta_{y}(s)} .
$$

Justification of (4) thus amounts to showing that if $\alpha(s)$, $\beta(s)$ and $\gamma(s)$ are polynomials satisfying (3), then there must exist polynomials $\delta_{u}(s)$ and $\delta_{y}(s)$ such that (11) holds where $\delta_{u}(s) / \gamma(s)$ and $\delta_{y}(s) / \gamma(s)$ are strictly proper and proper transfer functions respectively. The following proposition implies this and more.

Proposition 1: Let $\alpha, \beta, \gamma$ be fixed monic polynomials with $\alpha$ and $\beta$ coprime and $\alpha / \beta$ proper; and let $n$ and $n^{*}$ be fixed positive integers with $n \geqslant n^{*}$. For each nonzero constant $g_{p}$ and each pair of monic and coprime polynomials $\alpha_{p}$ and $\beta_{p}$ satisfying

$$
\left(\alpha_{p} / \beta_{p}\right)^{\circ}=n^{*}
$$

and

$$
\left(\beta_{p}\right)^{\circ} \leqslant n
$$

there exist polynomials $\delta_{y}$ and $\delta_{u}$ satisfying

$$
\begin{aligned}
& \left(\delta_{u} / \gamma\right)^{\circ}>0 \\
& \left(\delta_{y} / \gamma\right)^{\circ} \geqslant 0
\end{aligned}
$$

and (11), if and only if $\alpha$ divides $\gamma$,

$$
(\alpha / \beta)^{\circ}=n^{*},
$$

and

$$
(\gamma)^{\circ} \geqslant n-1
$$

If $\alpha, \beta$ and $\gamma$ have the required properties and if the inequality in (15) is strict, then there exist $\delta_{u}$ and $\delta_{y}$ satisfying (11) and (13) for which the inequality in (13b) is strict.

A proof of this proposition appears in the Appendix.

The proposition clearly implies that in order to be able to select polynomials $\alpha, \beta$, and $\gamma$ for which (4) is guaranteed to hold, it is not only sufficient to know $n^{*}$ and $n$, but necessary as well. Thus, even though process model assumptions 2b) and 2c) may appear somewhat severe, they are unavoidable consequences of any approach based on system representation (4). Whether or not one can design an adaptive system under weaker hypotheses (e.g., by only assuming bounds for $n^{*}$ are known, rather than $n^{*}$ itself) is very much an open matter.

Remark 1: It is of some interest to know whether or not a particular selection of $\alpha, \beta$, and $\gamma$ provides a "minimal parameterization," i.e., a parameterization in which $\delta_{u}, \delta_{y}$, and consequently $k$ are uniquely determined by $g_{p}, \alpha_{p}$, and $\beta_{p}$. It can be shown that if $\alpha, \beta$, and $\gamma$ are chosen to satisfy (14), (15), and the hypotheses of Proposition 1, then for any pair of coprime, monic polynomials $\alpha$ and $\beta_{p}$ satisfying (12) there exist unique polynomials $\delta_{u}$ and $\delta_{y}$, satisfying (13) provided: 1) $\alpha$ and $\alpha_{p}$ are coprime, and 2) either $\gamma^{\circ}=\left(\beta_{p}\right)^{\circ}-1$ or $\gamma^{\circ}=\left(\beta_{p}\right)^{\circ}$ and (13b) is required to hold with inequality. While coprimeness of $\alpha$ and $\alpha_{p}$ can be guaranteed by simply choosing $\alpha=1$, it is clearly not possible to choose $\gamma$ to satisfy 2 ) above, unless, of course, if $\left(\beta_{p}\right)^{\circ}$ is known exactly.

\section{State-Space Interpretation}

Before concluding this section we briefly outline an alternative approach leading to (9), based on state-space considerations. This approach, which was what originally led us to (9), provides further insight by characterizing (9) as an estimate of a desired state-feedback control law. For simplicity, we outline the approach under the assumption that the polynomials $\alpha(s), \beta(s)$, and $\gamma(s)$ satisfying (3) have been selected so that $\alpha(s)=1,(\beta(s))^{\circ}=n^{*}$ and $(\gamma(s))^{\circ}=n$; we further assume that $T_{r}(s)=g_{r} / \beta(s)$, where $g_{r}$ is a constant.

The approach is based on two easily proved facts: First, if $\left(c_{1 \times n}, A_{n \times n}\right)$ is any observable pair, with $\gamma(s)$ the characteristic polynomial of $A$, then there exist $n$-vectors $h_{p}$ and $b_{p}$ such that

$$
c\left(s I-A-h_{p} c\right)^{-1} b_{p}=\frac{\alpha_{p}(s)}{\beta_{p}(s)} .
$$

Second, for at least one pair $\left(h_{p}, b_{p}\right)$ satisfying $(16)$, there exists a row vector $f_{p}$ such that $A+h_{p} c+b_{p} g_{p} f_{p}$ is stable and

$$
c\left(s I-A-h_{p} c-b_{p} g_{p} f_{p}\right)^{-1} b_{p}=\frac{1}{\beta(s)} .
$$

Equation (16) implies that

$$
\left.\begin{array}{l}
\dot{x}_{p}(t)=\left(A+h_{p} c\right) x_{p}(t)+b_{p} g_{p} u(t) \\
y(t)=c x_{p}(t)
\end{array}\right\}
$$

is a valid process model. Since $\dot{x}_{p}$ can also be written as

$$
\dot{x}_{p}(t)=\left(A+h_{p} c+b_{p} g_{p} f_{p}\right) x_{p}(t)+b_{p} g_{p}\left(u(t)-f_{p} x_{p}(t)\right)
$$


and since $A+h_{p} c+b_{p} g_{p} f_{p}$ is stable, we can use (17) to obtain

$$
y(t)=\frac{g_{p}}{\beta(s)}\left(u(t)-f_{p} x_{p}(t)\right) \quad(\epsilon)
$$

By assumption, $y_{r}(t)=\left(g_{r} / \beta(s)\right) r(t)$, so the output tracking error can now be written as

$$
e(t)=\frac{g_{p}}{\beta(s)}\left(u(t)-f_{p} x_{p}(t)-\left(g_{r} / g_{p}\right) r(t)\right) \quad(\epsilon)
$$

This suggests that desired system behavior might be achieved by setting $u(t)=\hat{f}(t) \hat{x}(t)+\hat{g}(t) r(t)$ where $\hat{f}(t), \hat{x}(t)$ and $\hat{g}(t)$ are suitably defined estimates of $f_{p}, x_{p}(t)$ and $g_{r} / g_{p}$, respectively-but as mentioned at the beginning of this section, this approach leads to difficult problems which so far have not been overcome.

The alternative approach pursued here is to use an estimate of the product $f_{p} x_{p}(t)$ rather than the product of estimates of $f_{p}$ and $x_{p}(t)$, respectively. The structure of the proposed estimate is motivated by the fact that the state $x_{p}(t)$ of system (18) can be written as

$$
x_{p}(t)=E_{u}(t) b_{p} g_{p}+E_{y}(t) h_{p}+e^{A t} q
$$

where $E_{u}(t)$ and $E_{y}(t)$ are any pair of solutions to the matrix differential equations

$$
\left.\begin{array}{l}
\dot{E}_{u}(t)=A E_{u}(t)+I u(t) \\
\dot{E}_{y}(t)=A E_{y}(t)+I y(t)
\end{array}\right\} .
$$

$I$ is the $n \times n$ identity and $q$ is a constant vector depending on the initial values of $x_{p}, E_{u}$, and $E_{y}$ [10]. Since $A$ is a stable matrix by hypothesis, it follows from (20) that

$$
f_{p} x_{p}(t)=f_{p} E_{u}(t) b_{p} g_{p}+f_{p} E_{y}(t) h_{p} \quad(\epsilon) .
$$

Using just (21), (22), and the stability of $A$, it is not difficult to verify that $f_{p} x_{p}(t)$ can also be written as

$$
f_{p} x_{p}(t)=\sum_{i=1}^{2 n} \theta_{i}(t) k_{i} \quad(\epsilon)
$$

where as before the $k_{i}$ are unknown constants and the $\theta_{i}(t)$ are scalar-valued sensitivity functions obtained by passing $u$ and $y$ through stable linear filters. In view of (23) it is now clear that the sum

$$
\sum_{i=1}^{2 n} \theta_{i}(t) \hat{k}_{i}(t)+g_{r} r(t) \hat{k}_{(2 n+1)}(t)
$$

will yield an estimate of $f_{p} x_{p}(t)+\left(g_{r} / g_{p}\right) r(t)$ provided $\hat{k}_{i}(t)$ is an estimate of $k_{i}$ for $i \in\{1,2, \cdots, 2 n\}$ and $k_{(2 n+1)}(t)$ is an estimate of $1 / g_{p}$.

The preceding development shows, at least under the special assumptions on $\alpha, \beta, \gamma$, and $T_{r}(s)$, that the term $\theta^{\prime}(t) \hat{k}(t)$ appearing in (9) is really an estimate of $f_{p} x_{p}(t)+$ $\left(g_{r} / g_{p}\right) r(t)$ where $f_{p} x_{p}(t)$ is a state feedback law, which if applied to process model (18), would have the effect of shifting the model's poles from the root set of $\beta_{p}(s)$ to the root set of $\alpha_{p}(s) \beta(s)$. The more general situation, when $\alpha$, $\beta, \gamma$, and $T_{r}(s)$ are not constrained by these special assumptions, admits a similar state-space interpretation, provided system (18) is replaced with a more general linear model, representing the process together with a dynamic compensator.

\section{Control Equations}

To complete our description of the proposed adaptive system, we first select $\alpha(s)$ and $\beta(s)$ so that (3) holds, and in addition so that $\beta(s)=\beta^{*}(s) \delta(s)$, where $\beta^{*}(s)$ is monic and $\alpha(s) / \beta^{*}(s)$ is a strictly proper, strictly positive real transfer function. It is easy to check that these constraints imply that $\delta(s)$ must be a monic, stable polynomial of degree $n^{*}-1$.

It turns out in what follows that nothing essential is lost if $\alpha(s) / \beta^{*}(s)$ is assumed to be the transfer function of a one-dimensional system, i.e., $\alpha(s) / \beta^{*}(s)=1 /\left(s+\lambda_{0}\right)$ for some $\lambda_{0}>0$. For the sake of simplicity we henceforth make this assumption; the more general situation in which $\alpha(s) / \beta^{*}(s)$ is the positive real transfer function of a higher dimensional system can be treated along similar lines using the Kalman-Yakubovich lemma.

These assumptions together with (5) and the control law defined by (9) imply that the output tracking error $e(t)$ can be written as

$$
e(t)=\frac{1}{\left(s+\lambda_{0}\right) \delta(s)} g_{p}\left(\theta^{\prime}(t) \bar{k}(t)+\bar{u}(t)\right) \quad(\epsilon)
$$

where $\bar{k}(t)$ is the parameter error

$$
\bar{k}(t)=\hat{k}(t)-k
$$

We wish to develop a parameter adjustment law for $\hat{k}(t)$ and an expression for $\bar{u}(t)$. Since these equations are considerably more complicated for the case $n^{*}>1$ than for the case $n^{*}=1$, these cases will be treated separately.

\section{Special Case $n^{*}=1$}

The crucial consequence of the hypothesis $n^{*}=1$ is that $\delta(s)=1$, implying that the transfer function $1 /((s+$ $\left.\lambda_{0}\right) \delta(s)$ ) appearing in (24) is positive real. The importance of this is that if we set $\bar{u}(t)=0$ and

$$
\Sigma_{\hat{k}}: \dot{\hat{k}}(t)=-\left(\operatorname{sign}\left(g_{p}\right)\right) Q \theta(t) e(t),
$$

where $Q$ is any prespecified, positive definite, constant gain matrix, then for any reference input $r(t)$, bounded for $t \geqslant 0$, and for any initial time $t_{0} \geqslant 0$ and state $x_{\Sigma}\left(t_{0}\right)$, the state response $x_{\Sigma}(t) \equiv\left(x_{p}(t), x_{r}(t), x_{\theta}(t), \hat{k}(t)\right)$ of the resulting closed-loop adaptive system $\Sigma$ described by (1), (2), (5), (8), (9), and (26) exists for $t \geqslant t_{0}$ and is bounded uniformly in $t_{0}$. To understand why this is so, first observe that we can now use (24)-(26) to write

$$
\dot{e}(t)=-\lambda_{0} e(t)+g_{p}\left(\theta^{\prime}(t) \bar{k}(t)\right)+\epsilon(t)
$$




$$
\dot{\bar{k}}(t)=-\left(\operatorname{sign}\left(g_{p}\right)\right) Q \theta(t) e(t)
$$

where $\epsilon(t)$ is a linear combination of strictly decaying exponentials. Next observe that since $\Sigma$ is smooth dynamical system, for any initial time $t_{0} \geqslant 0$ and any initial state $x_{\Sigma}^{0}$, there must be a nonempty interval $\left[t_{0}, T\right)$ of maximal length on which $x_{\Sigma}(t)$ exists. If the derivative of the nonnegative time function

$$
V(t)=\frac{1}{2}\left(e^{2}(t)+\left|g_{p}\right| \bar{k}^{\prime}(t) Q^{-1} \bar{k}(t)+\frac{1}{2 \lambda_{0}} \int_{t}^{\infty} \epsilon^{2}(\tau) d \tau\right)
$$

is evaluated on $\left[t_{0}, T\right)$ there results from (27)

$$
\dot{V}(t)=-\left(\sqrt{\lambda_{0}} e(t)-\frac{1}{2 \sqrt{\lambda_{0}}} \epsilon(t)\right)^{2} \quad t \in \epsilon\left(t_{0}, T\right) .
$$

Since $\dot{V} \leqslant 0$ and $V \geqslant 0$, it must be that $0 \leqslant V(t) \leqslant V\left(t_{0}\right)$; this and (28) clearly show that $e(t)$ and $\bar{k}(t)$ are bounded on $\left[t_{0}, T\right)$ by a constant not depending on $t_{0}$ or $T$. Boundedness of $\bar{k}(t)$ and (25) imply boundedness of $\hat{k}(t)$, whereas boundedness of $r(t)$ for $t \geqslant 0$ and stability of $\Sigma_{r}$ imply boundedness of $x_{r}(t)$; the latter together with boundedness of $e(t)$ and (5) imply boundedness of $y(t)$. To prove that $x_{p}(t)$ is bounded, it is therefore enough to establish boundedness of $u(t)$; in view of (9) and the hypothesis $\bar{u}(t)=0, u(t)$ will be bounded provided $\theta(t)$ is. Since both $r(t)$ and $y(t)$ are bounded and since (8) is a stable system, it follows that $\theta(t)$ [and $x_{\theta}(t)$ ] will be bounded provided $\left(s I-A_{\theta}\right)^{-1} b_{u} u(t)$ is; but $\left(s I-A_{\theta}\right)^{-1} b_{u} u(t)=((s I-$ $\left.\left.A_{\theta}\right)^{-1} b_{u}(s+1)\right)(1 /(s+1)) u(t)(\epsilon)$ and $\left.s I-A_{\theta}\right)^{-1} b_{u}(s+1)$ is a proper transfer matrix. To establish boundedness of $\left(s I-A_{\theta}\right)^{-1} b_{u} u(t)$ it is therefore enough to show that $(1 /(s$ $+1)) u(t)$ is bounded. To do this we make explicit use of process model assumption 1) and the hypothesis $n^{*}=1$ to conclude that $(1 /(s+1))\left(T_{p}(s)\right)^{-1}$ is a stable, proper transfer function. Since $(1 /(s+1)) u(t)=(1 /(s+1))\left(T_{p}(s)\right)^{-1} y(t)$ $(\epsilon)$, and since $y(t)$ is bounded, it follows that $(1 /(s+$ 1)) $u(t)$ is bounded as well.

The preceding argument shows that $x_{\Sigma}(t)$ exists and is bounded on $\left[t_{0}, T\right)$ by a constant not depending on $t_{0}$ or $T$. The latter property together with the smoothness of $\Sigma$ ensure that $x_{\Sigma}(t)$ can be extended to $[T, \infty)$ so that for all $t \in\left[t_{0}, \infty\right), x_{\Sigma}(t)$ satisfies $\Sigma$ 's state equation and is bounded uniformly in $t_{0}$.

To show that $e(t) \rightarrow 0$ as $t \rightarrow \infty$ we first use (28) and (29) to conclude that $V(t)$ is a monotone nonincreasing function of $t$; since, in addition, $V(t) \geqslant 0$, it must be that $V_{\infty} \equiv \lim _{t \rightarrow \infty} V(t)<\infty$. This shows that the integral $\int_{t_{0}}^{\infty} \dot{V}(\tau) d \tau$ converges. Since, in addition, (29) and (27) imply that both $\dot{V}(t)$ and $\ddot{V}(t)$ are bounded on $\left[t_{0}, \infty\right)$, it follows that $\lim _{t \rightarrow \infty} \dot{V}(t)=0$, and hence with (29) that $e(t) \rightarrow 0$ as $t \rightarrow \infty$. The following theorem summarizes what has been established thus far.

Theorem I: If $n^{*}=1$, if $r(t)$ is any reference input, bounded and piecewise-continuous on $[0, \infty)$, and if $\Sigma$ is the closed-loop adaptive system described by (1), (2), (5), (8), (9), (26), and $\bar{u}(t)=0$, then for arbitrary initial time $t_{0} \geqslant 0$ and initial state $x_{\Sigma}^{0}$ and for all $t \geqslant t_{0}$, the state response $x_{\Sigma}\left(t, t_{0}, x_{\Sigma}^{0}\right)$ of $\Sigma$ exists and is uniformly bounded in $t_{0}$. In addition, along any such state response, the system's output tracking error satisfies

$$
\lim _{t \rightarrow \infty} e(t)=0 .
$$

\section{General Case $n^{*}>1$}

For the remainder of this paper we focus attention on the more general and considerably more difficult case when $n^{*}>1$. Since $\delta(s)$ is now necessarily of positive degree, implying that the transfer function $1 /\left(\left(s+\lambda_{0}\right) \delta(s)\right)$ in (24) is no longer positive real, to proceed it becomes necessary to introduce auxiliary filters of much the same structure as those first proposed by Monopoli [7]. These filters are described by the equations

$$
\Sigma_{H}:\left\{\begin{array}{l}
\dot{H}(t)=A H(t)+b \theta^{\prime}(t) \\
\phi^{\prime}(t)=c H(t)
\end{array}\right.
$$

and

$$
\Sigma_{x}: \dot{x}(t)=A x(t)-b \vec{u}(t)+H(t) \dot{\hat{k}}(t)
$$

where $(c, A, b)$ is an $\left(n^{*}-1\right)$-dimensional realization of $1 / \delta(s), H(t)$ is an $\left(n^{*}-1\right) \times\left(2 n_{\gamma}+2\right)$ matrix, and $x(t)$ is an $\left(n^{*}-1\right)$-vector; specific equations for $\hat{k}(t)$ will be given in a moment.

Using (24), (30), (31), and the assumed stability of $A$, it is straightforward to verify that

$$
e(t)=\frac{1}{\left(s+\lambda_{0}\right)}\left(g_{p}\left(\phi^{\prime}(t) \bar{k}(t)-c x(t)\right)\right)
$$

Thus, if $\bar{e}(t)$ is an auxiliary error defined by the equations

$$
\Sigma_{x_{0}}:\left\{\begin{array}{l}
\dot{x}_{0}(t)=-\lambda_{0} x_{0}(t)+\hat{g}(t) c x(t) \\
\bar{e}(t)=x_{0}(t)+e(t)
\end{array}\right.
$$

where $\hat{g}(t)$ is a signal intended to estimate $g_{p}$, then it is possible to write

$$
\dot{\bar{e}}(t)=-\lambda_{0} \bar{e}(t)+g_{p} \phi^{\prime}(t) \bar{k}(t)+c x(t) \bar{g}(t)+\epsilon(t)
$$

where

$$
\bar{g}(t)=\hat{g}(t)-g_{p} .
$$

and $\epsilon(t)$ is a linear combination of strictly decaying exponential time functions. The significance of (33) is that it has much the same structure as (27a). This suggests that system stability might be achieved by setting $\bar{u}(t)=0$,

$$
\Sigma_{\hat{k}}: \dot{\hat{k}}(t)=-\left(\operatorname{sign}\left(g_{p}\right)\right) Q \phi(t) \bar{e}(t)
$$

and

$$
\Sigma_{\dot{g}}: \dot{\hat{g}}(t)=-q c x(t) \bar{e}(t)
$$


where $Q$ is any preselected positive definite, constant, gain matrix, and $q$ is an arbitrary positive constant. Indeed, for any choice of $\bar{u}(t),(35)$ and (36) imply that

$$
\begin{aligned}
& \dot{\bar{k}}(t)=-\left(\operatorname{sign}\left(g_{p}\right)\right) Q \phi(t) \bar{e}(t) \\
& \dot{\bar{g}}(t)=-q c x(t) \bar{e}(t)
\end{aligned}
$$

and thus with (33) that the time derivative of the nonnegative function

$$
\begin{aligned}
V(t)=\frac{1}{2}\left(\bar{e}^{2}(t)+\left|g_{p}\right| \bar{k}^{\prime}(t) Q^{-1} \bar{k}(t)\right. & +\frac{1}{q} \bar{g}^{2}(t) \\
& \left.+\frac{1}{2 \lambda_{0}} \int_{t}^{\infty} \epsilon^{2}(\tau) d \tau\right)
\end{aligned}
$$

along any solution to differential equations describing the overall adaptive system is

$$
\dot{V}(t)=-\left(\sqrt{\lambda_{0}} \bar{e}(t)-\frac{1}{2 \sqrt{\lambda_{0}}} \epsilon(t)\right)^{2}
$$

For the same reasons as before, it can be concluded from (39) and (40) that $\bar{e}(t), \bar{k}(t)$, and $\bar{g}(t)$ are bounded time functions on some interval $\left[t_{0}, T\right)$ of finite length; however, from these two equations and (32) it is not possible to conclude that $e(t)$ is bounded since there is no guarantee that $c x(t)$ is. Indeed one can construct an example with $\bar{u}(t)=$ 0 , for which $c x(t)$ is not bounded assuming $x(t)$ is a solution to (31) [8]. However, since this example uses a contrived $H(t)$ rather than one known to satisfy (30a), the example does not really demonstrate system instability. On the other hand, the example does show that much more elaborate arguments involving the equations which generate both $H(t)$ and $\theta(t)$ are required to prove boundedness, at least for the case $\bar{u}(t)=0$. Although we have recently obtained results suggesting that the adaptive system obtained by setting $\bar{u}(t)=0$ [with $\hat{k}(t)$ and $\hat{g}(t)$ as defined in (35) and (36)] actually has a bounded state response for any bounded reference input [12], this issue is not yet settled.

To achieve a bounded state response, we shall continue to use the parameter adjustment laws for $\hat{k}(t)$ and $\hat{g}(t)$ as given by (35) and (36), but we shall no longer take $\bar{u}(t)$ to be zero. Instead we shall set

$$
u=f(H) x
$$

where $f(H)$ is a row-vector of polynomial functions of the elements of $H ; f(\cdot)$ and is defined as follows. With

$$
\bar{n} \equiv n^{*}-1
$$

and $P_{0}$ any preselected $\bar{n} \times \bar{n}$ positive definite matrix, write $P$ for the unique positive definite solution to the equation

$$
P A+A^{\prime} P=-P_{0} .
$$

For $i \in\{1, \cdots, \bar{n}\}$, let $M_{i}$ denote the $i \times i$ matrix

$$
\left.\begin{array}{r}
M_{1}=\left(C_{1} P^{-1} C_{1}^{\prime}\right)^{-1} \\
M_{i}=\left(C_{i} P^{-1} C_{i}^{\prime}\right)^{-1}\left(I_{i \times i}-\sum_{j=1}^{i-1} C_{i} P^{-1} C_{j}^{\prime} M_{j} E_{j, i}\right), \\
i \in\{2, \cdots, \bar{n}\}
\end{array}\right\}
$$

where $I_{i \times i}$ is the $i \times i$ identity,

$$
C_{i} \equiv\left[c^{\prime}, A^{\prime} c^{\prime}, \cdots,\left(A^{\prime}\right)^{i-1} c^{\prime}\right]^{\prime}, \quad i \in\{1,2, \cdots, \bar{n}\}
$$

and

$$
E_{j, i}=\left[I_{j \times j}, 0\right]_{j \times i}, \quad 0<j \leqslant i .
$$

Next, let $g_{i}\left(C_{i} H\right)$ and $w_{i}^{\prime}\left(C_{i} H\right)$ be $i$-component row vectors defined by the formulas

$$
\begin{gathered}
w_{1}^{\prime}=-\left(c H Q H^{\prime} c^{\prime}\right)\left(\operatorname{sign}\left(g_{p}\right)\right) \\
g_{1}=-c N_{1} \\
w_{i}^{\prime}=\left[w_{i-1}^{\prime},-\left(\left(c A^{i-1} H Q H^{\prime} c^{\prime}\right)\left(\operatorname{sign}\left(g_{p}\right)\right)+g_{i-1} w_{i-1}\right)\right] \\
i \in\{2, \cdots, \bar{n}\} \\
g_{i}=\sum_{j=1}^{2\left(n_{\mathrm{r}}+1\right)} h_{i, j}^{\prime} R_{i}^{\prime} \frac{\partial g_{i-1}}{\partial h_{i-1, j}} E_{i-1, i}+g_{i-1} R_{i} \\
-\left(c A^{i-1}+g_{i-1} C_{i-1}\right) N_{i}, \quad i \in\{2, \cdots, \bar{n}\}
\end{gathered}
$$

where

$$
N_{i}=P^{-1} \sum_{j=1}^{i} C_{j}^{\prime} M_{j} w_{j} w_{j}^{\prime} M_{j}^{\prime} E_{j, i}
$$

and

$$
R_{i+1}=\left[0, I_{i \times i}\right]_{i \times(i+1)} ;
$$

$h_{i, j}$ is the $j$ th column of $C_{i} H$ and $\partial g_{i-1} / \partial h_{i-1, j}$ is the $(i-1) \times j$ matrix of partial derivatives of $g_{i-1}\left(C_{i-1} H\right)$ with respect to the elements of $h_{i-1, j}$. It is important to note that these definitions imply that $g_{i}, w_{i}$, and hence $N_{i}$ are matrices of polynomial functions of the elements of $C_{i} H$.

Next observe that because of the structure of matrices $E_{i, j}$ defined by (45), the $\bar{n} \times \bar{n}$ matrix

$$
G=I+\left[\begin{array}{c}
0 \\
g_{1} E_{1, \bar{n}} \\
g_{2} E_{2, \bar{n}} \\
\vdots \\
g_{\bar{n}-1} E_{\bar{n}-1, \bar{n}}
\end{array}\right]
$$

is lower triangular, with ones on its diagonal. From this and the aforementioned function dependence of $g_{i}$ on $C_{i} H$, it follows that $G^{-1}$ exists and that the elements of 
both $G$ and $G^{-1}$ are polynomial functions of the elements of $H$. Hence, if $f$ is now defined as

$$
f=c A^{\bar{n}}-\left(c A^{\bar{n}}+g_{n} C_{n}\right)\left(\left(C_{n}\right)^{-1} G C_{n}\right)^{-1}
$$

then $f$ is a row-vector of polynomials in the elements of $H .^{2}$ Our specific reason for defining $f$ in this way will become apparent in the next section.

To summarize, the proposed adaptive controller for the case $n^{*}>1$ consists of the reference system $\Sigma_{r}$ defined by (2), the output tracking error (5), the sensitivity function system $\Sigma_{\theta}$ defined by (8), the control law (9), the filtered sensitivity function system $\Sigma_{H}$ defined by (30), the auxiliary error system $\Sigma_{x_{0}}$ defined by (32), the parameter adjustment laws (35) and (36), and the system

$$
\Sigma_{x}: \dot{x}=(A-b f(H)) x-H Q H^{\prime} c^{\prime} \bar{e}\left(\operatorname{sign}\left(g_{p}\right)\right)
$$

which results when the expressions for $\dot{\hat{k}}, \phi$, and $\bar{u}$ given by (35), (30b), and (41) respectively, are substituted into (31). Careful examination of the equations involved reveals that the resulting adaptive control system consisting of $\Sigma_{r}, \Sigma_{p}, \Sigma_{x_{0}}, \Sigma_{\theta}, \Sigma_{H}, \Sigma_{\hat{g}}, \Sigma_{\dot{k}}$, and $\Sigma_{x}$ is a well-posed dynamical system in that within each feedback loop, there is a strictly proper transfer function.

\section{SyStem Stability}

Our main result is as follows.

Theorem 2: If $n^{*}>1$, if $r(t)$ is any reference input, bounded, and piecewise continuous on $[0, \infty)$, and if $\Sigma$ is the closed-loop adaptive system described by (1), (2), (5), (8), (9), (30), (32), (35), (36), and (54), then for arbitrary initial time $t_{0} \geqslant 0$ and initial state $x_{\Sigma}^{0}$ and for all $t \geqslant t_{0}$, the state response $x_{\Sigma}\left(t, t_{0}, x_{\Sigma}^{0}\right)$ of $\Sigma$ exists and is bounded uniformly in $t_{0}$. In addition, along any such state response,

$$
\begin{aligned}
& \lim _{t \rightarrow \infty} e(t) \rightarrow 0 \\
& \lim _{t \rightarrow \infty} x(t) \rightarrow 0 \\
& \lim _{t \rightarrow \infty} x_{0}(t) \rightarrow 0 .
\end{aligned}
$$

The adaptive system $\Sigma$ can be viewed as a dynamical system of the form $\dot{x}_{\Sigma}=\mu\left(x_{\Sigma}, r\right)$, where $x_{\Sigma}$ is a composite state vector consisting of subvectors $\hat{k}, \hat{g}, x_{p}, x_{r}, x_{\theta}, H, x$, and $x_{0}$, and $\mu$ is a continuous function of $x_{\Sigma}$ and $r$. Theorem 2 clearly implies for any fixed, bounded $r(t)$, that the zero solution to the differential equation $\dot{\bar{x}}=$ $\mu(\bar{x}, r(t))-\mu\left(\bar{x}_{0}(t), r(t)\right)$ is globally, uniformly stable, $\bar{x}_{0}(t)$ being the (bounded) state response of $\Sigma$ to $r(t)$ assuming a zero initial state at time $t_{0}=0$. It is in this sense that $\Sigma$ can be characterized as a Lyapunov stable adaptive system.

In view of the preceding theorem and the structure of $\bar{u}$ as defined by (41), it is clear that $\bar{u}(t) \rightarrow 0$ as $t \rightarrow \infty$. On the other hand, it cannot be concluded from Theorem 2 (or Theorem 1) that the parameter errors $\bar{k}(t)$ and $\bar{g}(t)$ tend to

\footnotetext{
${ }^{2}$ Note that $C_{\bar{n}}$ is nonsingular because $(c, A)$ is observable.
}

zero as $t \rightarrow \infty$. In fact, these errors can be expected to go to zero only if the process model on which controller design is based is minimally parameterized (see Remark 1 ). If this is the case and if $r(t)$ is in some sense "persistently exciting," then it is at least plausible that both $\bar{k}(t)$ and $\bar{g}(t)$ will asymptotically approach zero. It appears likely that this can be shown to be so without too much difficulty using (for example) the results of [11].

The proof of Theorem 2 depends on six lemmas. The first describes certain algebraic relationships which exist between a positive-definite matrix $P$ and matrices $M_{i}$ defined by (43).

\section{Lemma 1:}

$$
P=\sum_{j=1}^{n} C_{j}^{\prime} M_{j} C_{j}
$$

and

$$
C_{i} P^{-1} C_{j}^{\prime} M_{j} C_{j}=0, \quad j>i>0 .
$$

Proof: The definition of $M_{i}$ in (43) implies that

$$
I_{i \times i}=\sum_{k=1}^{i} C_{i} P^{-1} C_{k}^{\prime} M_{k} E_{k, i}, \quad i \in\{1,2, \cdots, \bar{n}\} .
$$

If both sides of this equation are postmultiplied by $C_{i}$ and if $C_{k}$ is then substituted for $E_{k, i} C_{i}$, there results

$$
C_{i}=\sum_{k=1}^{i} C_{i} P^{-1} C_{k}^{\prime} M_{k} C_{k}, \quad i \in\{1,2, \cdots, \pi\}
$$

Equation (55) is obtained by premultiplying each side of (57), at $i=\bar{n}$, by $P\left(C_{\bar{n}}\right)^{-1}$.

To establish (56), replace $i$ with $j$ in (57) so that

$$
C_{j}=\sum_{k=1}^{j} C_{j} P^{-1} C_{k}^{\prime} M_{k} C_{k}, \quad j \in\{1,2, \cdots, \bar{n}\} .
$$

If, for $j>i$, both sides of this equation are premultiplied by $E_{i, j}$ and if $C_{i}$ is then substituted for $E_{i, j} C_{j}$, there results

$$
C_{i}=\sum_{k=1}^{j} C_{i} P^{-1} C_{k}^{\prime} M_{k} C_{k}, \quad j>i \geqslant 1 .
$$

Subtraction from (57) thus yields

$$
0=\sum_{k=i+1}^{j} C_{i} P^{-1} C_{k}^{\prime} M_{k} C_{k}, \quad j>i \geqslant 1
$$

from which (56) follows.

The next lemma is a simple consequence of (30) and of the definition of $(c, A, b)$.

Lemma 2:

$$
\begin{aligned}
\phi^{\prime} & =C_{1} H \\
C_{i} \dot{H} & =R_{i+1} C_{i+1} H \quad i \in\{1,2, \cdots, \bar{n}-1\} \\
c A^{\bar{n}-1} \dot{H} & =c A^{\bar{n}} H+\theta^{\prime}
\end{aligned}
$$

Proof: Equation (58a) is a direct consequence of 
(30b) and (44). Since $(c, A, b)$ canonically realizes $1 / \delta(s)$, it must be true that $c A^{\bar{n}-1} b=1$ and that $c A^{i-1} b=0, i \in$ $\{1, \cdots, \bar{n}-1\}$. The former and (30a) imply (58c), whereas the latter clearly show that $C_{i} b=0, i \in\{1, \cdots, \bar{n}-1\}$. Hence using (30a), $C_{i} \dot{H}=C_{i} A H, i \in\{1, \cdots, \bar{n}-1\}$; from these expressions and the identity $R_{i+1} C_{i+1}=C_{i} A$, it follows that $(58 \mathrm{~b})$ is true.

The following lemma describes the key algebraic relationships upon which our stability proof is based.

Lemma 3: If $S \equiv\left(C_{\bar{n}}\right)^{-1} G C_{\bar{n}}$ and

$$
z=S^{-1} x
$$

then

$$
c z=c x
$$

and

$$
\dot{z}=A z+w \bar{e}-P^{-1} \sum_{i=1}^{n} C_{i}^{\prime} M_{i} C_{i} w w^{\prime} C_{i}^{\prime} M_{i}^{\prime} C_{i} z
$$

where

$$
w=\left(C_{\bar{n}}\right)^{-1} w_{n}
$$

Proof: In view of (54) it is enough to show that

$$
\begin{aligned}
& c S=c \\
& S w=-H Q H^{\prime} c^{\prime}\left(\operatorname{sign}\left(g_{p}\right)\right)
\end{aligned}
$$

and

$$
\dot{S}+S(A-X)=(A-b f) S
$$

where

$$
X=P^{-1} \sum_{i=1}^{n} C_{i}^{\prime} M_{i} C_{i} w w^{\prime} C_{i}^{\prime} M_{i}^{\prime} C_{i}
$$

From (45) and (48), $w_{i}=E_{i, n} w_{n}, i \in\{1,2, \cdots, \bar{n}\}$; hence by (62), $w_{i}=E_{i, n} C_{n} w$. Since (44) and (45) provide the identities $C_{i}=E_{i, n} C_{n}, i \in\{1,2, \cdots, \bar{n}\}$, we can write

$$
w_{i}=C_{i} w, \quad i \in\{1,2, \cdots, \bar{n}\} .
$$

The definition of $G$ in (52) implies that $c$ is the first row of $G C_{n}$ and, for $i>1$, that $c A^{i-1}+g_{i} E_{i, n} C_{n}$ is the $i$ th row. Since $G C_{\bar{n}}=C_{n} S$, the $i$ th row of $C_{n} S$, namely $c A^{i-1} S$, must satisfy (63) for $i=1$ and $c A^{i-1} S=c A^{i-1}+$ $g_{i-1} E_{i-1, n} C_{n}$ for $i>1$; using the identity $C_{i-1}=E_{i-1, n} C_{n}$, $i \in\{2, \cdots, \bar{n}\}$, the latter can be written as

$$
c A^{i-1} S=c A^{i-1}+g_{i-1} C_{i-1}, \quad i \in\{2, \cdots, \bar{n}\} .
$$

Since $c=C_{1},(63)$ and (67) imply that $c S w=w_{1}$; from this and (46) it follows that the equation

$$
c A^{i-1} S w=-c A^{i-1} H Q H^{\prime} c^{\prime}\left(\operatorname{sign}\left(g_{p}\right)\right)
$$

holds for $i=1$. Now fix $i \in\{2, \cdots, \bar{n}\}$ and use (67) and (68) to obtain $c A^{i-1} S w=c A^{i-1} w+g_{i-1} w_{i-1}$. Note that $c A^{i-1} w$ is the $i$ th row of $C_{i} w$ which by (67) is the $i$ th row of $w_{i}$. Thus $c A^{i-1} S w$ equals $g_{i-1} w_{i-1}$ plus the $i$ th row of $w_{i}$. From this and the definition of $w_{i}$ in (48) it follows that (69) is true for this value of $i$.

The preceding argument proves that (69) holds for $i \in\{1, \cdots, \bar{n}\}$. Since $(c, A)$ is an observable pair, we have shown that (64) is true.

Next consider the expression

$$
N_{i} C_{i}=P^{-1} \sum_{j=1}^{i} C_{j}^{\prime} M_{j} C_{j} w w^{\prime} C_{j}^{\prime} M_{j}^{\prime} C_{j}, \quad i \in\{1,2, \cdots, \bar{n}\}
$$

which is what results if both sides of (50) are postmultiplied by $C_{i}$ and if $C_{j}$ and $C_{j} w$ are then substituted for $E_{j, i} C_{i}$, and $w_{j}$, respectively. In view of (56) and the definition of $X$ in (66), it follows that

$$
C_{i} N_{i} C_{i}=C_{i} X, \quad i \in\{1,2, \cdots, \bar{n}\} .
$$

Since $C_{1}=c$, (70) together with the definition of $g_{1}$ in (47) imply that $g_{1} C_{1}=-c X$. Hence by (68), $c A S=c A-c X$. This and (63) thus allow us to write

$$
c A S=c \dot{S}+c S(A-X) .
$$

To develop similar expressions for $c A^{i} S, i>1$, we first use the identity $\left[g_{i-1}, 1\right] C_{i}=c A^{i-1}+g_{i-1} C_{i-1}, i \in\{2, \cdots$, $\bar{n}\}$, together with (68) and (70) to obtain

$$
\left(c A^{i-1}+g_{i-1} C_{i-1}\right) N_{i} C_{i}=c A^{i-1} S X, \quad i \in\{2, \cdots, \bar{n}\} .
$$

Next we exploit the fact that for $i \in\{2, \cdots, \bar{n}\}, g_{i-1}$ depends only on $C_{i-1} H$. This enables us to write

$$
\dot{g}_{i-1}=\sum_{j=1}^{2\left(n_{\gamma}+1\right)} \dot{h}_{i-1, j}^{\prime} \frac{\partial g_{i}}{\partial h_{i-1, j}}, \quad i \in\{2, \cdots, \bar{n}\}
$$

where $h_{i-1, j}$ is the $j$ th column of $C_{i-1} H$. Using (58b) which implies that $\dot{h}_{i-1, j}=R_{i} h_{i, j}, i \in\{2, \cdots, \bar{n}\}$, we can write

$$
\dot{g}_{i-1}=\sum_{j=1}^{2\left(n_{\gamma}+1\right)} h_{i, j}^{\prime} R_{i}^{\prime} \frac{\partial g_{i-1}}{\partial h_{i-1, j}}, \quad i \in\{2, \cdots, \bar{n}\} .
$$

Since $E_{t-1, i} C_{i}=C_{i-1}$, it follows that

$$
\dot{g}_{i-1} C_{i-1}=\sum_{j=1}^{2\left(n_{\gamma}+1\right)} h_{i, j}^{r} R_{i}^{\prime} \frac{\partial g_{i-1}}{\partial h_{i-1, j}} E_{i-1, i} C_{i} .
$$

If for $i \in\{2, \cdots, \bar{n}\}$ the expression defining $g_{i}$ in (49) is postmultiplied by $C_{i}$ and if (72) and (73) are then substituted into the result, one obtains the expression

$$
g_{i} C_{i}=\dot{g}_{i-1} C_{i-1}+g_{i-1} R_{i} C_{i}-c A^{i-1} S X, \quad i \in\{2, \cdots, \bar{n}\} .
$$

Addition of $c A^{i}$ to both sides, followed by substitutions of $C_{i-1} A$ for $R_{i} C_{i}, c A^{i-1} S$ for $c A^{i-1}+g_{i-1} C_{i-1}$, and $c A^{i-1} \dot{S}$ for $\dot{g}_{i-1} C_{i-1}$ yields

$$
c A^{i}+g_{i} C_{i}=c A^{i-1}(\dot{S}+S(A-X)), \quad i \in\{2, \cdots, \bar{n}\} .
$$


For $i \in\{2, \cdots, \bar{n}-1\},(68)$ implies that the left side of (74) can be replaced by $c A^{i} S$. This together with (71) allows us to write

$$
c A^{i} S=c A^{i-1}(\dot{S}+S(A-X)), \quad i \in\{1, \cdots, \bar{n}-1\} .
$$

As noted in the proof of Lemma $2, c A^{i-1} b=0$, iE $\{1, \cdots, \bar{n}-1\}$; thus the preceding expression can be written as

$$
\begin{aligned}
c A^{i-1}(A-b f) S=c A^{i-1}(\dot{S}+S(A-X)) & \\
& i \in\{1, \cdots, \bar{n}-1\} .
\end{aligned}
$$

The definition of $f$ in (53) implies that $f S+c A^{\bar{n}}+g_{\bar{n}} C_{\bar{n}}$ $=c A^{\bar{n}} S$. Since $c A^{\bar{n}} b=1$, we can write $c A^{\bar{n}-1}(A-b f) S=$ $c A^{\bar{n}}+g_{\bar{n}} C_{\bar{n}}$. From this and (74), it is now clear that (75) also holds for $i=\bar{n}$. Since $(c, A)$ is observable, it follows from (75) that (65) is true.

\section{Lemma 4:}

$$
C_{i} \dot{z}=R_{i+1} C_{i+1} z+w_{i} \bar{e}-C_{i} N_{i} E_{i, i+1} C_{i+1} z, \quad i \in\{1, \cdots, \bar{n}-1\} .
$$

Proof: From (61), (66), and (70) it follows that

$$
C_{i} \dot{z}=C_{i} A z+C_{i} w \bar{e}-C_{i} N_{i} C_{i} z, \quad i \in\{1, \cdots, \bar{n}-1\} .
$$

To obtain the desired result, substitute $R_{i+1} C_{i+1}$ for $C_{i} A$, $E_{i, i+1} C_{i+1}$ for $C_{i}$, and use (67) to replace $C_{i} w$ by $w_{i}$.

Lemma 5: It is possible to write

$$
e^{(i)}=\mu_{i}\left(\bar{e}, \vec{k}, \bar{g}, x_{0}, C_{i} H, C_{i} z, \epsilon_{i}\right), \quad i \in\{1, \cdots, \bar{n}\}
$$

where $e^{(f)}$ is the ith derivative of $e, \epsilon_{i}$ is a linear combination of decaying exponential time functions, and $\mu_{i}$ is a continuous function of its arguments.

Proof: Observe from (32b) that $e=\bar{e}-x_{0}$. If this expression is differentiated, if (32a) and (33) are used to eliminate derivatives, and if (34), (58a), and (60) are then used to eliminate $\hat{g}, \phi$, and $c x$, the resulting expression is $\dot{e}=\mu_{1}\left(\bar{e}, \bar{k}, \bar{g}, x_{0}, C_{1} H, C_{1} z, \epsilon_{1}\right)$ where $\mu_{1}$ is an infinitely differentiable function. If this expression is, in turn, differentiated, and if (32)-(34), (37), (38), Lemma 2, and Lemma 4 are used to express $\ddot{e}$ in terms of $\bar{e}, \bar{k}, \bar{g}, x_{0}, C_{2} H, C_{2} z, \epsilon_{2}$, $w_{1}\left(C_{1} H\right)$, and $N_{1}\left(C_{1} H\right)$, then there results $\ddot{e}=$ $\mu_{2}\left(\bar{e}, \bar{k}, \bar{g}, x_{0}, C_{2} H, C_{2} z, \epsilon_{2}\right)$ where $\mu_{2}$ is an infinitely differentiable function of its arguments. Repeating this process and making use of the fact that $w_{i}$ and $N_{i}$ depend only on $C_{i} H$, one obtains a sequence of infinitely differentiable (and hence continuous) functions $\mu_{i}$ for which (76) is true.

Lemma 6: There exist proper, stable transfer matrices $\bar{T}(s), T_{0}(s), \cdots, T_{\bar{n}}(s)$ such that

$$
c A^{i} H=\left(\bar{T}(s) e^{(i)}+T_{i}(s) r\right)^{\prime} \quad(\epsilon), \quad i \in\{0, \cdots, \bar{n}-1\}
$$

and

$$
\theta^{\prime}=\left(\bar{T}(s) e^{(\bar{n})}+T_{n}(s) r\right)-c A^{\bar{n}} H \quad(\varepsilon)
$$

Proof: Definitions (8) and (30) of $\theta$ and $H$, respectively, imply that

$$
(c H)^{\prime}=\frac{1}{\delta}\left[\begin{array}{c}
T_{u} u \\
T_{y} y \\
\operatorname{Tr}
\end{array}\right]
$$

where $1 / \delta, T_{u}, T_{y}$, and $T$ are stable, proper transfer matrices. Since $T_{u}$ is actually strictly proper and since $(1 / \delta)^{\circ}=\left(T_{p}\right)^{\circ}-1$, it follows that $(1 / \delta)\left(T_{p}\right)^{-1} T_{u}$ is a proper transfer matrix; by process model assumption 1), $(1 / \delta)\left(T_{p}\right)^{-1} T_{u}$ is also stable. In addition, since $y=T_{p} u$ we can write $(1 / \delta) T_{u} u=(1 / \delta)\left(T_{p}\right)^{-1} T_{u} y(\epsilon)$. Substitution in (78) then yields

$$
(c H)^{\prime}=\bar{T} y+\left[\begin{array}{c}
0 \\
0 \\
(1 / \delta) T
\end{array}\right] r \quad(\epsilon)
$$

where $\bar{T} \equiv(1 / \delta)\left[\left(T_{p}\right)^{-1} T_{u}^{\prime}, T_{y}^{\prime}, 0\right]^{\prime}$ is a stable, proper transfer matrix. From (2) and (5), $y=e+T_{r} r$; substitution in (79) thus provides

$$
(c H)^{\prime}=\bar{T} e+T_{0} r \quad(\epsilon)
$$

where $T_{0} \equiv \bar{T} T_{r}+[0,0,(1 / \delta) T]^{\prime}$. Since, by assumption, $T_{r}$ is a stable transfer matrix satisfying $\left(T_{r}\right)^{\circ} \geqslant\left(T_{p}\right)^{\circ}$, and since $\bar{n}=\left(T_{p}\right)^{\circ}-1$, it follows that $T_{0}$ is a matrix of proper, stable transfer functions, each of relative degree no smaller than $\bar{n}$. Thus if $T_{i}(s)$ is now defined to be $s^{i} T_{i}(s)$, $i \in\{1, \cdots, \bar{n}\}$, then $T_{i}$ must be stable and proper and by (80), the $i$ th derivative of $c H$, written $c H^{(i)}$, must satisfy

$$
c H^{(i)}=\bar{T} e^{(i)}+T_{i} r \quad(\epsilon) \quad i \in\{1, \cdots, \bar{n}\} .
$$

But Lemma 2 implies that $c H^{(i)}=c A^{i} H, i \in\{0, \cdots, \bar{n}-1\}$ and that $c H^{(\bar{n})}=\theta^{\prime}+c A^{\bar{n}} H$. From these relations (80) and (81) it now follows that the lemma is true.

Proof of Theorem 2: Let $r(t), t_{0}>0$ and $x_{\Sigma}^{0}$ be fixed. The smoothness of $\Sigma$ implies that for some $T>t_{0}$, the state response $x_{\Sigma}(t)$ exists on $\left[t_{0}, T\right)$. With $\epsilon(t)$ as in (33), let $V(t)$ denote the nonnegative time function

$$
\begin{aligned}
V=\frac{1}{2}\left(\bar{e}^{2}+\left|g_{p}\right| \bar{k}^{\prime} Q^{-1} \bar{k}\right. & +\frac{1}{q} \bar{g}^{2} \\
& \left.+\frac{1}{\lambda_{0}} \int_{t}^{\infty} \epsilon^{2}(\tau) d \tau\right)+\frac{\lambda_{0}}{\bar{n}} z^{\prime} P z .
\end{aligned}
$$

Differentiation of $V$, elimination of resulting derivatives using (33), (37), (38), (61), completion of the square in $\bar{e}$ and $\epsilon$, and substitution of $P_{0}$ for $-\left(P A+A^{\prime} P\right)$ yields

$$
\begin{aligned}
\dot{V}=-\frac{1}{2}\left(\sqrt{\lambda_{0}}\right. & \left.\bar{e}-\frac{1}{\sqrt{\lambda_{0}}} \epsilon\right)^{2}-\frac{\lambda_{0}}{\bar{n}} z^{\prime} P_{0} z \\
& -\frac{2 \lambda_{0}}{\bar{n}}\left(\frac{\bar{n}}{4} \bar{e}^{2}-z^{\prime} P w \bar{e}+\sum_{i=1}^{n}\left(z^{\prime} C_{i}^{\prime} M_{i} C_{i} w\right)^{2}\right) .
\end{aligned}
$$

Using (55) to eliminate $P$ from the preceding expression, 
followed by completion of squares, provides

$$
\begin{aligned}
\dot{V}=-\frac{1}{2}\left(\sqrt{\lambda_{0}} \bar{e}-\frac{1}{\sqrt{\lambda_{0}}} \epsilon\right)^{2}-\frac{\lambda_{0}}{\bar{n}} z^{\prime} P_{0} z \\
-\frac{2 \lambda_{0}}{\bar{n}} \sum_{i=1}^{n}\left(\frac{\bar{e}}{2}-z^{\prime} C_{i} M_{i} C_{i} w\right)^{2} .
\end{aligned}
$$

Equations (82) and (83) clearly imply that $V, \bar{e}, \bar{k}, \bar{g}$, and $z$ are bounded on $\left[t_{0}, T\right)$ by a constant not depending on $t_{0}$ or $T$. It will now be shown in six steps that the eight constituents $\hat{k}, \hat{g}, x_{0}, H, x, x_{r}, x_{p}, x_{\theta}$ of the state response of $\Sigma$ are each bounded on $\left[t_{0}, T\right.$.

1) Boundedness of $\hat{k}$ and $\hat{g}$ are direct consequences of (25) and (34), respectively, together with the boundedness of $\bar{k}$ and $\bar{g}$, respectively.

2) Boundedness of $x_{0}$-Boundedness of $z$ and (60) imply that $c x$ is bounded; from this (32a) and the boundedness of $\hat{g}$ it follows that $x_{0}$ is bounded.

3) Boundedness of $H$-Boundedness of $\bar{e}$ and $x_{0}$ together with (32b) imply boundedness of $e$; since $r$ is also bounded, it follows from (77a) that $c H$ (i.e., $C_{1} H$ ) is bounded. If for fixed $i<\bar{n}, e^{(i-1)}$ and $C_{i} H$ are bounded, then by Lemma $5 e^{(i)}$ is bounded, and thus by Lemma 6 $c A^{i} H$ and hence $C_{i+1} H$ are also bounded. By induction, $C_{\bar{n}} H$ and thus $H$ are bounded.

4) Boundedness of $x-$ By Lemma $3, x=S z$. Since $S$ depends continuously on $H$ and since both $H$ and $z$ are bounded, it follows that $x$ is bounded.

5) Boundedness of $x_{r}$ and $x_{p}$-Boundedness of $r$ and stability of $\Sigma_{r}$ clearly imply boundedness of $x_{r}$. This together with (5) and boundedness of $e$ imply boundedness of $y$. Since $\Sigma_{p}$ is a canonical system with a bounded output, to establish boundedness of $x_{p}$ it is enough to show that $u$ is bounded. For this, first observe that boundedness of $H$ together with Lemma 5 imply boundedness of $e^{(\bar{n})}$; thus by (77b), $\theta$ is bounded. Next observe that (9) and (41) imply that $u$ depends continuously on $\hat{k}, \theta, \bar{e}, x$, and $f$; since $\hat{k}, \theta, \bar{e}$, and $x$ are bounded and since $f$ depends continuously on $H$, which is also bounded, it follows that $u$ and therefore $x_{p}$ are bounded as well.

6) Boundedness of $x_{\theta}$ is a consequence of the stability of $\Sigma_{\theta}$ and the boundedness of $u, y$, and $r$.

The preceding arguments prove that $x_{\Sigma}(t)$ is bounded on $\left[t_{0}, T\right)$ by a constant not depending on $t_{0}$ or $T$. From this and the smoothness of $\Sigma$ it follows that $x_{\Sigma}(t)$ can be extended to $[T, \infty)$ so that for all $t \geqslant t_{0}, x_{\Sigma}(t)$ satisfies $\Sigma$ 's state equations and is bounded uniformly in $t_{0}$.

To prove that $e, x$, and $x_{0}$ approach zero as $t \rightarrow \infty$, it will first be shown that $\dot{V}(t) \rightarrow 0$ as $t \rightarrow \infty$. For this, observe from (83) that $\dot{V} \leqslant 0$; thus $V(t)$ is a monotone nonincreasing function of $t$, bounded below by zero. This implies that $\lim _{t \rightarrow \infty} V(t)<\infty$ and thus that the integral $\int_{t_{0}}^{\infty} \dot{V}(t) d t$ converges. Therefore $\dot{V}(t)$ will approach zero provided $\dot{V}(t)$ and $\ddot{V}(t)$ are both bounded for $t \geqslant t_{0}$; since (83) together with the continuous dependence of $w$ on $H$ [i.e., $\left.w=C_{n} w_{n}\left(C_{n} H\right)\right]$ and the already established boundedness of $\bar{e}, z$, and $H$ clearly imply the former, it is enough to establish the latter. Differentiation of the expression in (83) shows that $\ddot{V}$ is a continuous function of the quantities $\bar{e}, \epsilon, w, z, \dot{\bar{e}}, \dot{\epsilon}, \dot{w}$, and $\dot{z}$. Boundedness of $\dot{\bar{e}}$ follows from (33) together with the boundedness of $\bar{e}, \bar{k}, x, \bar{g}, \epsilon$, and $\phi^{\prime}(=c H)$. Boundedness of $\dot{\epsilon}$ is obvious, since $\epsilon$ is a linear combination of decaying exponentials. Recall that $w_{\bar{n}}$ is a polynomial function of the components of $H$; since $w=C_{n} w_{n}$, this and boundedness of $H$ and $\dot{H}$ (which is a consequence of (30a) and boundedness of $\theta$ ) imply boundedness of $\dot{w}$. Boundedness of $\dot{z}$ follows from (61) and the boundedness of $z, w$, and $\bar{e}$. Thus $\ddot{V}(t)$ is bounded for $t \geqslant t_{0}$.

The preceding argument proves that $\dot{V}(t) \rightarrow 0$ as $t \rightarrow \infty$. Examination of (83) reveals that this can occur only if $\bar{e}$ and $z$ approach zero as $t \rightarrow \infty$. Since from (59) $x=S z$ and since $S$ depends continuously on $H$, it follows that $S$ is bounded and thus that $x(t) \rightarrow 0$ as $t \rightarrow \infty$. This together with (32a) and the boundedness of $\hat{g}$ imply that $x_{0}(t) \rightarrow 0$ as $t \rightarrow \infty$. It now follows from (32b) that $e(t) \rightarrow 0$ as $t \rightarrow \infty$.

\section{CONCLUDING REMARKS}

In this paper we have outlined a procedure for designing a parameter-adaptive controller capable of causing the output of a process to approach and track the output of a prespecified reference model with zero steady-state error. While the controller is admittedly complex, to our knowledge it is the only differentiator-free dynamical adaptive controller proposed thus far which, without making unnecessarily restrictive process model assumptions, has been shown to provide globally stable closed-loop operation when applied to a single-input, single-output linear system. Whether or not global stability can also be achieved with other, possibly less complex adaptive controllers (e.g., the one considered in this paper with $\bar{u}=0$ ) is an interesting question for further study.

The principal result of this paper-that it is actually possible to adaptively control a linear system with zero steady-state tracking error--is contrary to our earlier expectations [10]. The reservations expressed in [10] are prompted by the observation that with certain adaptive configurations, unbounded controller gains might result as current estimates of process transfer function coefficients approach values for which the estimating transfer function has a pole and zero in common. What this paper shows is that this problem can be avoided by applying to the process a control signal which, in effect, is an estimate of the product of desired feedback gains and process state rather than the product of distinct estimates of desired feedback gains and process state. At present, the generation of such a control signal seems possible only for those desired feedback laws which, if applied to the process, would cause all process transfer function zeros to be cancelled. For this reason, it is not clear if the possibility of unbounded gains can be avoided in those situations where "adaptive-zero cancelling" cannot be employed 
(i.e., when the process transfer function has right-half plane zeros.)

Since Theorems 1 and 2 are true, independent of the stability of the open-loop process model, the results presented here are potentially applicable to the problem of identifying process models not assumed to be open-loop stable. Recent simulation experiments have shown, that for such models, the overall set of differential equations describing the adaptive system can be quite sensitive to computer roundoff errors unless the initial parameter errors are small. Such problems, which we believe to be characteristic of all parameter-adaptive algorithms, can apparently be minimized by appropriately choosing the various gains associated with the adaptive algorithm. Systematic techniques for selecting these gains to reduce sensitivity or to increase convergence rates are obviously needed, but so far have not been developed.

\section{APPENDIX}

\section{Proof of Proposition 1}

Sufficiency: Suppose that $\alpha, \beta$, and $\gamma$ satisfy (14) and (15) with $\alpha$ dividing $\gamma$. Write $\sigma$ and $\rho$ for the unique quotient and remainder of $\beta \gamma$ divided by $\alpha \beta_{p}$; thus

$$
\beta \gamma=\alpha \beta_{p} \sigma+\rho
$$

where $\rho^{\circ}<\left(\alpha \beta_{p}\right)^{\circ}$. Since by hypothesis, $\alpha$ divides $\gamma$, it follows from (A1) that $\alpha$ divides $\rho$; thus $\rho=\alpha \bar{\rho}$ where $\bar{\rho}^{\circ}<\left(\beta_{p}\right)^{\circ}$. If $\delta_{y}$ is now defined so that $\delta_{y}=-\left(1 / g_{p}\right) \bar{\rho}$, then

$$
\beta \gamma=\alpha \beta_{p} \sigma-g_{p} \delta_{y} \alpha
$$

and $\left(\delta_{y}\right)^{\circ}<\left(\beta_{p}\right)^{\circ}$. The latter together with (12b) and (15) ensure that $\delta_{y}$ satisfies (13b); and if (15) is a strict inequality, then so is $(13 b)$.

Since $\rho^{\circ}<\left(\alpha \beta_{p}\right)^{\circ}$, (A1) implies $\left(\alpha \beta_{p} \sigma\right)^{\circ}=(\beta \gamma)^{\circ}$; thus $\left(\alpha \beta_{p} \sigma \alpha_{p}\right)^{\circ}=\left(\beta \gamma \alpha_{p}\right)^{\circ}$. In addition, since $(12 a)$ and (14) imply $\left(\beta \alpha_{p}\right)^{\circ}=\left(\beta_{p} \alpha\right)^{\circ}$, it follows that $\left(\alpha \beta_{p} \sigma \alpha_{p}\right)^{\circ}=\left(\gamma \beta_{p} \alpha\right)^{\circ}$; hence, $\left(\sigma \alpha_{\rho}\right)^{\circ}=\gamma^{\circ}$. Since (A1) also implies that $\sigma$ is monic and thus that $\sigma \alpha_{p}$ is monic, if $\delta_{u}$ is defined as

$$
\delta_{u}=\gamma-\sigma \alpha_{p}
$$

then (13a) must be true. Elimination of $\sigma$ from (A2) and (A3) now yields (11) which is the desired result.

Necessity: Let $\alpha_{p}$ and $\beta_{p}$ be polynomials satisfying the proposition's hypotheses with $\alpha_{p}$ and $\alpha$ coprime and $\left(\beta_{p}\right)^{\circ}$ $=n$; such polynomials clearly exist. Suppose that $\delta_{y}$ and $\delta_{u}$ are polynomials satisfying (11) and (13). Since $\alpha$ and $\alpha_{p}$ are coprime, it follows from (11) that $\alpha$ divides $\gamma \beta$; but $\alpha$ and $\beta$ are coprime by hypothesis, so $\alpha$ must divide $\gamma$ as the Proposition asserts.

Since $\alpha_{p}$ and $\alpha \beta_{p}$ are coprime, (11) will hold provided

$$
\sigma \alpha_{p}=\gamma-\delta_{u}
$$

and

$$
\sigma \alpha \beta_{p}=\gamma \beta+g_{p} \alpha \delta_{y}
$$

for some polynomial $\sigma$. From (A4) and (13a) it follows that

$$
\gamma^{\circ}=\left(\sigma \alpha_{p}\right)^{\circ} \text {. }
$$

By hypothesis, both $\alpha / \beta$ and $\delta_{y} / \gamma$ are proper; hence $(\gamma \beta)^{\circ} \geqslant\left(\gamma \beta+g_{p} \alpha \delta_{y}\right)^{\circ}$. This and (A5) imply $(\gamma \beta)^{\circ} \geqslant$ $\left(\sigma \alpha \beta_{p}\right)^{\circ}$; but from the relation $\left(\sigma \alpha \beta_{p}\right)^{\circ}=\left(\sigma \alpha \beta_{p} \alpha_{p}\right)^{\circ}-\left(\alpha_{p}\right)^{\circ}$ and (A6) there follows $\left(\sigma \alpha \beta_{p}\right)^{\circ} \geqslant\left(\gamma \alpha \beta_{p}\right)^{\circ}-\left(\alpha_{p}\right)^{\circ}$. Thus $(\gamma \beta)^{\circ} \geqslant\left(\gamma \alpha \beta_{p}\right)^{\circ}-\left(\alpha_{p}\right)^{\circ}$. This and (12a) imply that $\beta^{\circ} \geqslant \alpha^{\circ}$ $+n^{*}$; but $n^{*}>0$, so $\beta^{\circ}>\alpha^{\circ}$. From this, (A5), and (13b), it now follows that

$$
\left(\sigma \alpha \beta_{p}\right)^{\circ}=(\gamma \beta)^{\circ} .
$$

Substitution of (A6) in (A7) and elimination of common factors yields $\left(\alpha \beta_{p}\right)^{\circ}=\left(\alpha_{p} \beta\right)^{\circ}$; from this and (12a), it now follows that (14) is true.

Since (15) must necessarily hold for $n=1$, assume $n \geqslant 2$ and suppose (15) is false. Then $\gamma^{\circ} \leqslant n-2$; since $\left(\beta_{p}\right)^{\circ}=n$,

$$
\left(\beta_{p}\right)^{\circ}-\gamma^{\circ} \geqslant 2 \text {. }
$$

From (12a) and (14), $\beta^{\circ}=\left(\beta_{p}\right)^{\circ}+\alpha^{\circ}-\left(\alpha_{p}\right)^{\circ}$. Since (A6) implies $\left(\alpha_{p}\right)^{\circ} \leqslant \gamma^{\circ}$, it follows that

$$
\beta^{\circ} \geqslant\left(\beta_{p}\right)^{\circ}+\alpha^{\circ}-\gamma^{\circ} .
$$

Choose $\bar{\rho}$ so that

$$
\bar{\rho}^{\circ}=\alpha^{\circ}+1
$$

and so that $\beta-\bar{\rho}$ has a root as $s=0$. Since (A8) and (A9) imply $\beta^{\circ} \geqslant \alpha^{\circ}+2$, it follows from (A10) that $\beta^{\circ}>\bar{\rho}^{\circ}$; hence $(\beta-\bar{\rho})^{\circ}=\beta^{\circ}$. Therefore, from (A9) $(\beta-\bar{\rho})^{\circ} \geqslant\left(\beta_{p}\right)^{\circ}$ $+\alpha^{\circ}-\gamma^{\circ}$. Since $(\beta-\bar{\rho})$ has a real root, it follows that there is a factorization

$$
\beta-\bar{\rho}=\bar{\sigma} \theta
$$

where $\theta$ is a monic factor satisfying

$$
\theta^{\circ}=\left(\beta_{p}\right)^{\circ}+\alpha^{\circ}-\gamma^{\circ} \text {. }
$$

From this and (A8), there follows $\theta^{\circ} \geqslant \alpha^{\circ}+2$. Therefore from (A10) $\theta^{\circ}>\bar{\rho}^{\circ}$. This and (A11) can be true only if $\bar{\rho}$ is the unique remainder of least degree of $\beta$ divided by $\theta$.

Since $\alpha$ divides $\gamma$, there must be a polynomial $\vec{\beta}_{p}$ such that $\alpha \bar{\beta}_{p}=\gamma \theta$. Since (A12) implies that $\left(\bar{\beta}_{p}\right)^{\circ}=\left(\beta_{p}\right)^{\circ}$ and since $\bar{\beta}_{p}$ is defined independent of $\beta_{p}$, we can assume that $\beta_{p}$ was selected at the outset so that $\beta_{p}=\bar{\beta}_{p}$. Thus $\alpha \beta_{p}=$ $\gamma \theta$. Hence from (A5), $\sigma \gamma \theta=\gamma \beta+g_{p} \alpha \delta_{y}$. Thus we may write

$$
\beta=\theta \sigma+\tilde{\rho}
$$

where $\tilde{\rho}$ is a polynomial satisfying

$$
\tilde{\rho} \gamma=-g_{p} \alpha \delta_{y} .
$$

Since $\bar{\rho}$ was shown to be the unique remainder of least degree of $\beta$ divided by $\theta$, it follows from (A13) that 
$\tilde{\rho}^{\circ} \geqslant \bar{\rho}^{\circ}$. This and (A14) imply that $\alpha^{\circ}+\left(\delta_{y}\right)^{\circ} \geqslant \gamma^{\circ}+\bar{\rho}^{\circ}$. Hence from (A10) $\alpha^{\circ}+\left(\delta_{y}\right)^{\circ} \geqslant \gamma^{\circ}+\alpha^{\circ}+1$; thus $\left(\delta_{y}\right)^{\circ} \geqslant \gamma^{\circ}$ +1 which contradicts (13b). Hence (15) is true.

\section{REFERENCES}

[1] R. E. Kalman "Desion of a self optimizing control system," Trans. ASME, vol. 80, pp. 468-478, Jan. 1958.

[2] W. Everleigh, Adaptive Control and Optimization Techniques. New York: McGraw-Hill, 1967.

[3] P. C. Parks, "Liapunov redesign of model reference adaptive control systems," IEEE Trans. Automat. Contr., vol. AC-11, pp. 362-367, July 1966.

[4] K. J. Aström and B. Wittenmark, "On self-tuning regulators," Automatica, vol. 9, pp. 185-199, 1973.

[5] L. Ljung and B. Wittenmark, "On a stabilizing property of adaptive regulators," Preprints, IFAC Symp. on Identification and Syst. Parameter Estimation, Tbilisi, USSR, 1976.

[6] L. Ljung, "On positive real transfer functions and the convergence of some recursive schemes," IEEE Trans. Automat. Contr., vol. AC-22, pp. 539-552, Aug. 1977.

[7] R. V. Monopoli, "Model reference adaptive control with an augmented error signal," IEEE Trans. Automat. Contr., vol. AC-19, pp. 474-484, Oct. 1974.

[8] A. Feuer, B. R. Barmish, and A. S. Morse, "An unstable dynamical system associated with model reference adaptive control," IEEE Trans Automat Contr, vol. AC-23, pp. 499-500, June 1978.

[9] G. Lüders and K. S. Narendra, "An adaptive observer and identifier for linear systems," IEEE Trans. Automat. Contr., vol. AC-18, pp. 496-499, Oct. 1973.

[10] A. S. Morse, "Representation and parameter identification of multi-output linear systems," in Proc. IEEE Conf. on Decision and Contr., 1974, pp. 301-306.

[11] A. P. Morgan and K. S. Narendra "On the stability of nonautonomous differential equations $\dot{x}=[A+B(t)] x$ with skew symmetric matrix $B(t)$," SIAM J. Contr., vol. 15, pp. 163-176, Jan. 1977.

[12] A. Feuer and A. S. Morse, "Local stability of parameter-adaptive control systems," in Proc. 1978 Conf. on Informa. Sci. and Syst., Johns Hopkins Univ., Baltimore, MD, Mar. 1978.

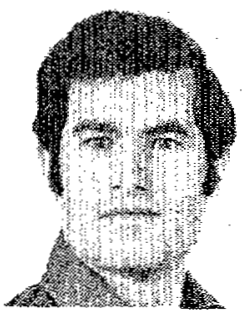

Arie Feuer (M'76) was born in Budapest Hungary, in September 1943. He received the B.Sc. degree in 1967 and the M.Sc. degree in 1973, both from the Technion-Israel Institute of Technology, Haifa.

From 1967 to 1970 he was associated with Technomatic-Israel, working on industrial automatization. He is currently completing his Ph.D. work in the area of adaptive control in the Department of Engineering and Applied Science, Yale University, New Haven, CT.

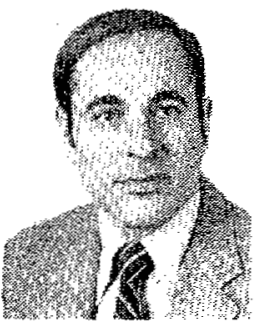

A. Stephen Morse (S'62-M'67-SM'78) was born in Mt. Vernon, NY, on June 18, 1939. He received the B.S.E.E. degree from Cornell University, Ithaca, NY, in 1962, the M.S. degree from the University of Arizona, Tucson, in 1964, and the $\mathrm{Ph}$.D. degree from Purdue University, Lafayette, IN, in 1967, all in electrical engineering.

From 1967 to 1970 he was associated with the Office of Control Theory and Application, NASA Electronics Research Center, Cambridge MA. Since July 1970 he has been at Yale University, New Haven, CT, where he is currently Professor of Engineering and Applied Science. His main interest is in system theory, and he has done research in network synthesis, optimal control, multivariable control, urban transportation, and adaptive control.

Dr. Morse has served as an Associate Editor of the IEEE TRANSACTIONS ON AUTOMATIC CONTROL, and as a Director of the American Automatic Control Council representing the Society for Industrial and Applied Mathematics. 